\title{
Laterality of Cerebral Controls on Somatic and Autonomic Functions
}

\author{
Hideo Tohgi, Yoshiyuki Kuroiwa, Toshiaki Konno and \\ Hitoshi Madarame \\ Department of Neurology, Iwate Medical University, \\ Morioka 020
}

Tohgi, H., Kuroiwa, Y., Konno, T and Madarame, H. Laterality of Cerebral Controls on Somatic and Autonomic Functions. Tohoku J. Exp. Med., 1990, 161, Suppl., 213-229 — The mode of cerebral representation for each half of the body is various according to the functions that are represented ; contralateral, ipsilateral and bilateral. We reviewed, and discussed the meaning of, such different patterns of cerebral representation for somatic and autonomic functions. cerebral representation; laterality ; fiber crossing

The laterality of cerebral representations for each half of the body in vertebrates is various according to the functions that are represented; contralateral, ipsilateral, and bilateral. Either one of the hemispheres may predominante over the other in controlling some functions. What determines, then, such different patterns of cerebral representation for different functions? This fundamental problem in neurology has attracted much interests of neuroscientists. The purpose of this paper is to review the various patterns in laterality of cerebral representations for somatic and autonomic functions, and to consider their meaning. Although textbooks of neurology and neuroanatomy describe many connections in the central nervous system, there still remains unsolved questions and controversies.

The search on cerebral representations for somatic and autonomic functions that are phylogenetically older than higher cerebral functions is also expected to contribute to our better understanding of cerebral representations for psychoneurological functions.

In this paper we will deal with the following systems ; (1) motor system, i.e., supranuclear, spinal and cranial motor; innervation and extrapyramidal system, (2) sensory system, and (3) autonomic nervous system, particularly in relation to pupillary contraction and urination.

\section{Motor system}

Pyramidal (cortico-spinal) tract

Although the major part of the pyramidal fibers crosses at the pyramidal 
decussation, a small proportion of the pyramidal fibers descends uncrossed. Some of the uncrossed fibers form the ventral corticospinal tract in the ventral funiculus of the cord along the ventral median fissure. This tract probably rarely descend below the level of the thoracic cord. Some of the uncrossed fibers join the crossed fibers of the lateral corticospinal tract. According to Hirasawa, crossed pyramidal fibers are the main pyramidal tract in mammals, while uncrossed fibers are accessory. The proportion of uncrossed pyramidal fibers is increased in phylogenetically higher mammals, being highest in man.

The ratio of uncrossed to crossed fibers varies individually. Nyberg-Hansen and Rinvik (1963) reviewed individual variations of the pyramidal tract in man, and described general norms for the fiber content of the pyramidal tract based upon the results of many investigators ; $75 \%$ of all pyramidal fibers course in the crossed lateral corticospinal tract, $10 \%$ in the lateral uncrossed tract and $15 \%$ in the ventral uncrossed tract.

The individual variation in the ratio of uncrossed to crossed fibers is of clinical importance, because uncrossed fibers may compensate the loss of crossed fibers. For example, hemiplegia due to capsular lesions may be milder in patients with higher proportion of uncrossed fibers. Kameyama et al. (1963) studied the relationship between the proportion of uncrossed fibers in the ventral corticospinal fibers to all pyramidal fibers and the clinical findings in autopsy patients with hemiparesis after stroke. They found that paradoxical motor symptoms such as ipsilateral or bilateral paresis, hyperreflexia, and Babinski's sign were frequently noted in patients in whom the ventral uncrossed fibers comprise more than $20 \%$ of all pyramidal fibers. In three cases hemiparesis was noted in the extremities ipsilateral to cerebral lesions. Conversely, a case who showed no degeneration of the ventral funiculus presented severe contralateral hemiplegia that did not recover at all.

Ipsilateral hemiplegia in cases with cerebral lesions confirmed by autopsy is extremely rare. Zenner (1898) described a case of brain tumor presenting ipsilateral hemiparesis: At autopsy, pyramidal decussation was absent. Since most cases reported as ipsilateral hemiparesis have been associated with brain tumors confirmed by operation, not by autopsy, the proportion of crossed and uncrossed fibers has not been determined. Therefore, ipsilateral hemiparesis reported in some cases with supratentorial brain tumors may have been due to Kernohan's notch.

Descending pathways from the cerebral cortex to cranial nerve motor nuclei Eye movement

Infranuclear fibers of the oculomotor nerve are mainly uncrossed, but partly crossed. The trochlear nerve is entirely crossed. The abducens nerve is entirely uncrossed. . Supranuclear controls are crossed to the superior rectus, uncrossed to the medial and inferior rectus and the inferior oblique, and bilateral to the levator palpebrae superioris. 
Efferent fibers for saccadic eye movements arise in area 8 (frontal eye field), descend anterior limb of the internal capsule, decussate at the midbrain, and terminate at the contralateral horizontal gaze center (PPRF) (Fig. 1). Thus, supranuclear innervation of saccadic eye movements is contralateral.

Efferent fibers for smooth pursuit movement originate from area 18 and 19, pass through posterior part of the posterior genu of the internal capsule, pulvinar, superior colliculus, and end at ipsilateral horizontal gaze center. They are supposed to cross twice, and to be virtually uncrossed. Therefore, supranuclear control of smooth pursuit movements is ipsilateral (Fig. 1).

Much of our knowledge of central oculomotor control have been inferred from clinico-pathological correlation in humans and from stimulation, recording, and ablation experiments in animals. However, anatomical pathways of cortical oculomotor control is still unclear.

Destruction of the frontal eye field or its descending pathway would be followed by a paralysis of conjugate ocular movements to the contralateral side. In stroke patients with corticofugal fibers interrupted in the internal capsule, conjugate deviation of the eyes to the side of lesion or impairment of conjugate voluntary movements to the side opposite the lesion occurs. This supranuclear control however is not absolute.

First, conjugate deviation or conjugate gaze palsy is usually transient, disappearing gradually in the course of recovery. Second, if the lesions as tumors

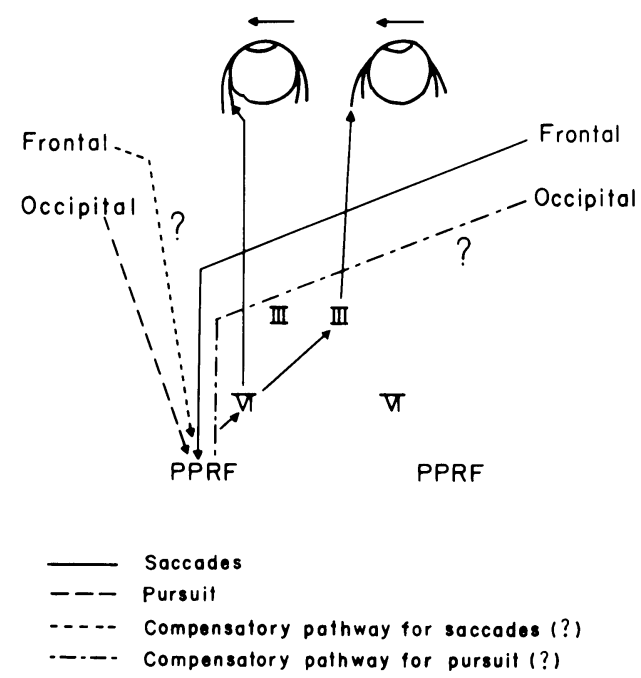

Fig. 1. Efferent fibers for saccadic eye movements (continuous line) arising in frontal eye field terminate at contralateral PPRF, while efferent fibers for smooth pursuit movements (broken line) originating from the occipital lobe end at ipsilateral PPRF. In case of destruction of these efferents, there may be compensatory ipsilateral frontal-PPRF and contralateral occipital-PPRF pathways. 
develop slowly, no symptom may occur at all. Brodal (1981) supposed some sort of compensation, presumably from the eye field of the other side.

In extremely rare instances, voluntary conjugate gaze is paralyzed completely and continues for a long period. Even in such cases, pursuing a slowly moving object is normally conducted. This phenomenon is termed fixation reflex by Holmes (1938).

A parieto-occipilal lesion impairs smooth pursuit movements to the side of lesion, giving rise to unilateral saccadic (cogwheel) pursuit, whereas impairment of saccadic movement due to a frontal lesion is to the side opposite to the lesion. Both saccadic palsy and smooth pursuit palsy are usually incomplete and transient. This necessitates quantitative measurement of ocular movement by nystagmography for an accurate estimation of supranuclear oculomotor disturbance.

In bilateral cerebral lesions, bilateral saccadic palsy generally occurs. When an old lesion is already present in either hemisphere, additional lesion in the opposite hemisphere gives rise to saccadic palsy that is enduring. Bilateral pursuit movement disorders with intact saccadic movements is a rare phenomenon, but has been reported in organic mercury poisoning. These clinical observations suggest bilateral cortical representations for horizontal gaze: Cerebral hemispheres compensate mutually in case of unilateral cerebral lesions.

\section{Eyelid movement}

Efferent fibers from the oculomotor nucleus are partly crossed and partly uncrossed. In the monkey, according to Warwick (1953), the medial and inferior recti and the inferior oblique muscles are supplied with uncrossed fibers only, the superior rectus receives only crossed fibers; while the levator palpebrae superioris has a bilateral innervation. This is in accordance with the difficulty in lifting both eyelids independently.

Neuroanatomy of supranuclear fibers to the levator nucleus of the oculomotor complex has not been established. Experimental stimulation of a number of cortical areas results in eyelid opening that is associated with other movements of the eye, head, and trunk. Cortical stimulation rarely exerts primary effect of lid opening. Cogan (1956) observed primary eyelid opening during stimulation of an area in the first frontal convolution just above the frontal eye fields. Eyelid elevation was greater on the side opposite the stimulation than the same side of the stimulus. In hemiplegic patients after stroke, according to our experience, the palpebral fissure on the hemiplegic side is often narrower than on the opposite side, if the orbicularis oculi muscle is not paralysed. Therefore, cerebral controls on eyelid elevation is bilateral, but contralateral innervation may slightly predominate.

Facial nerve

The facial nucelus presents a subdivision into several cell groups, corresponding to the innervation of certain facial muscles. In man, the oral muscles are 
supplied by a large lateral cell groups; the frontalis, corrugator supercilii, and orbicularis oculi muscles are innervated by a clearly delimited part of the nucleus.

There is difference in supranuclear innervation among these nuclei. The corticobulbar fibers descend crossed for the division for the lower two-thirds of the facial muscles, and both crossed and uncrossed for the upper one-third of the facial muscles. Supranuclear innervation to orbicularis oculi is predominantly crossed : It is possible to close either eye independently. If a hemiplegic patient is asked to close both eyes forcefully, eye closure on the hemiplegic side is insufficient; a sign called "signe de cil" in French literatures, or eye lash sign.

The supranuclear innervation for the frontal muscle, on the other hand, is symmetrical: We are unable to contract the right and left frontal muscles independently. The symmetrical control on the frontal muscle may be associated with its close relationship with conjugate upward movements of the eyes.

Glossopharyngeal and-vagal nerves

The special visceral efferent fibers, supplying the striated muscles of the pharynx and larynx and the upper part of the esophagus, arise from the nucleus ambiguus. Clinically, a unilateral lesion of the supranuclear tracts to the efferent vagus and glossopharyngeal nuclei is usually asymptomatic, while bilateral lesions give rise to severe impairment of pharyngeal and laryngeal movements (Haymaker 1969). This is the basis of our belief that the nucleus ambiguus receives bilateral and symmetrical corticobulbar fibers, partly crossed and partly uncrossed.

However, there are still problems open for reappraisal. First, patients with pseudobulbar palsy present dysarthria alone much more frequently than both dysarthria and dysphagia. Is there any difference in the topography of lesions between cases with dysarthria alone and cases with both dysarthria and dysphagia? Second, in our clinical practice, patients with unilateral cerebral lesions often become dysarthric. Does dysarthria occur in unilateral hemispheric lesions? If so, which part of the hemisphere would be involved in the production of dysarthria in such cases?

We studied a total of 142 patients (102 autopsy and 40 clinical cases) of cerebrovascular disease with dysarthria and/or dysphagia. The autopsy materials consisted of 40 cases with dysarthria alone and 62 cases with both dysarthria and dysphagia. Clinical cases had unilateral hemispheric lesions documented by $\mathrm{CT}$ scan, EEG, angiogram and routine neurologic examination.

In autopsy materials, the incidence of lesions in the pons, the internal capsule, basal ganglia, and the thalamus was compared between cases with dysarthria alone and cases with both dysarthria and dysphagia. The incidence of pontine lesions was significantly higher in cases with both dysarthria and dysphagia (45/ $62 ; 73 \%)$ than in cases with dysarthria alone $(20 / 40 ; 50 \%)(p<0.05)$. The difference in the incidence of bilateral pontine lesions between the two groups was more conspicuous ; $27 / 62(44 \%)$ for cases with both dysarthria and dysphagia, compared with $3 / 40(8 \%)$ for cases with dysarthria alone $(p<0.01)$. 
In cases with both dysarthria and dysphagia, the incidence of bilateral lesions was $21 / 62(34 \%)$ in the thalamus and in the posterior half of the putamen, while in cases with dysarthria alone the incidence of bilateral lesions in each of these areas was $6 / 40(15 \%)(p<0.05)$. Conversely, unilateral lesions in the anterior half of the putamen was significantly higher in cases with dysarthria alone (16/ $40,40 \%)$ than in cases with both dyarthria and dysphagia $(10 / 62,16 \%)(p<0.01)$.

These results suggest that dysphagia occurs not only with bilateral lesions in the pons, thalamus, and the posterior half of the putamen, but also with unilateral lesions in the anterior striatum.

If the materials were limited to the patients who had infarctions only in cerebral hemisheres, right hemiparesis was more frequent $(10 / 20,50 \%)$ than left hemiparesis $(4 / 20,20 \%)$ in cases with dysarthria alone, while right and left hemiparesis were found with equal frequencies $(4 / 7,23.5 \%)$ in cases with both dysarthria and dysphagia. Five cases with dysarthria alone showed unilateral cerebral lesions, 4 in the left hemisphere and one in the right. Three out of 4 cases with left hemisphere lesions had an infarction involving the anterior limb of the internal capsule, anterior striatum, and centrum semiovale adjacent to the anterior horn of the lateral ventricle, i.e. Wetterwinkel (watershed area).

In clinical materials, dysarthria was found in $26 \%(5 / 19)$ of patients with left hemiparesis, while in $67 \%(14 / 21)$ of patients with right hemiparesis $(p<0.05)$. Most dysarthric patients with left hemiparesis had large lesions in the right hemisphere due to occlusion of the internal carotid artery or middle cerebral artery, or putaminal hemorrhage. CT studies in five out of 14 dysarthric patients with right hemiparesis showed an infarction involving the anterior limb of the internal capsule, anterior striatum, and Wetterwinkel.

In conclusion, persistent dysarthria occurs with unilateral cerebral lesions, with higher frequency in left hemisphere lesions than in right hemisphere lesions. It is assumed that a lesion involving the internal capsule, anterior striatum, and Wetterwinkel in the left hemisphere interrupts corticobulbar fibers on the left side, and at the same time transects callosal fibers which transmit informations for articulatory movements to the right hemisphere, thus resulting in bilateral impairments of corticobulbar motor functions. It is also possible that an individual variation in the proportion of crossed and uncrossed corticobulbar fibers may contribute to the pathogenesis of dysarthria with unilateral cerebral lesions. If bilateral innervation of cortico-bulbar fibers from one hemisphere predominate over another, a lesion in the hemisphere that sends more cortico-bulbar fibers bilaterally than another hemisphere may give rise to dysarthria and dysphagia.

In an early period of acute stroke associated with unilateral lesion in the cerebral hemisphere, slight dysarthria and/or dysphagia may occur. Usually, dysarthria is far more frequent than dysphagia. In most cases dysarthria is transient, appearing in an early stage of the stroke and subsiding later. This may be that the nucleus ambiguus which normally receives supranuclear innervation 
from both hemispheres loses abruptly half of the input, resulting in bilateral weakness of muscles for articulation, and then gradually recovers by some sort of compensation.

In pseudo-bulbar palsy, dysarthria is far more frequent than dysphagia. Dysphagia, when it occurs, is almost always associated with dysarthria. In rare instances, however, definite dysphagia may be the only symptom with a lesion in cerebral hemisphere. Meadows (1973) reported such cases and concluded that the symptom is related to an affection of the lower part of the precentral gyrus.

\section{Accessory nerve}

Anatomically, the spinal portion of the accessory nucleus innervates the sternocleidomastoid and trapzius muscles (Netter 1958; Carpenter 1972). Very few degenerated upper motor neuron fibers to the spinal accessory nucleus were observed in the two human cases (Kuypers 1958).

In patients with unilateral cerebral lesion and consequent cotralateral hemiplegia, conjugate deviation of the eyes is to the side of lesion (Landouzy's law), and turning of the head is also as a rule to the side of lesion (Prevost's law). With irritative lesions as in Jacksonian epileptic seizures, both eyes and head deviate opposite the side of lesion. These clinical facts suggest homolateral supranuclear control to the sternocleido-mastoid muscles.

Supranuclear innervation, however, to the accessory nucleus is controversial. Most standard textbooks in neurology support bilateral innervation or predominantly contralateral input (Brodal 1957, 1981 ; DeJong, 1970 ; Mayo Clinic 1976 ; Haymaker and Kulenbeck 1978). Brodal (1981) states, "In hemiplegias a contralateral paresis of the muscles supplied by the accessory nerve is a common finding, due to the practically total crossing of the cortico-bulbar fibers."

Exceptionally, a few held ipsilateral innervation (Peele, 1961 ; Haymaker and Kulenbeck 1978; Patten 1981). Patten (1981) described that supranuclear control to the accessory nucleus is homolateral for the sternocleidomastoid (SCM) muscle and contralateral for the trapezius muscle.

Balegura and Katz (1980) studied stroke patients with hemiplegia on this account. They showed that $70 \%$ of cases who could barely elevate the arm against gravity were noted to have decreased power of the sternocleidomastoid muscle ipsilateral to the lesions.

We studied this problem on 64 hemiplegic patients after stroke. The muscle power was evaluated by the method of Medical Research Council. In patients with grade 3 or less muscle power of the upper extremity of the paretic side, sternocleidomastoid (SCM) muscle was paretic contralaterally to the lesion in $15 \%$ and ipsilaterally in $41 \%$ of the cases. Muscle power of the SCM was normal in $44 \%$ of the cases. In patients with grade 4 or more muscle power of the upper extremity, muscle power of the SCM was normal in $70 \%$ of the cases: Weakness of the SCM was noted contralaterally in $13 \%$ and ipsilaterally in $17 \%$ of the cases.

Since head turning involves not only the SCM but also other muscles situated 
deep in the neck, accurate evaluation of muscle power of the SCM in isolation is very difficult. With this reservation, our conclusion is, firstly, that in about $40 \%$ of the cases supranuclear innervation of the SCM may be bilateral and almost symmetrical, because severe weakness in the upper extremity was associated with normal power of the SCM in these patients. Secondly, in more than $50 \%$ of the cases, supranuclear innervation is at least partly asymmetrical, ipsilateral control predominating over contralateral control by about three times.

\section{Hypoglossal nerve}

The hypoglossal nucleus is thought to receive cortico-bulbar fibers which are mostly crossed, because supranuclear lesions impairs protrusion of the tongue to the side contralateral to the lesion.

Extrapyramidal system

Extrapyramidal disorders also pose problems with regard to bilateral and unilateral controls of the basal ganglia.

Parkinsonism due to cerebrovascular disease

A large proportion of parkinsonism in old population in Japan is associated with cerebrovascular disease. Clinical characteristics of vascular parkinsonism as compared with Parkinson's disease are as follows.

As an initial symptom, gait disturbance not associated with tremor was noted more frequently in patients with vascular parkinsonism than in patients with Parkinson's disease. On the other hand, tremor was found from the beginning of the illness more frequently in patients with Parkinson's disease than in patients with vascular Parkinsonism.

At the time of clinical examination, tremor was noticed in nearly equal proportion of cases. However, the typical parkinsonian tremor comprising 5 to 6 $\mathrm{Hz}$, rhythmic oscillation was seen more frequently in patients with Parkinson's disease than in cases with vascular parkinsonism. The incidence of definitely appreciable rigidity was about the same in both groups. However, cogwheelings were more frequent in Parkinson's disease than in vascular parkinsonism, while rigidity of the plastic type was more frequent in cases with vascular parkinsonism.

What is particularly interesting in regard with the present problem is the laterality of symptoms. The incidence of patients in whom parkinsonian symptoms is asymmetrical is greater in Parkinson's disease than in cerebrovascular parkinsonism. Gait disturbance that may be called midline symptom usually develop late in the illness in Parkinson's disease, while it appears in an early period of the disease in vascular parkinsonism.

Since vascular lesions are relatively circumscribed, and vascular parkinsonism is the result of stepwise increase in the number of such circumscribed lesions, asymmetrical symptoms are expected to be more frequent in vascular parkinsonism than in degenerative Parkinson's disease.

Pathologically, the incidence of lacunes or small infarctions in the basal ganglia and the thalamus was studied, comparing controls, vascular parkinsonism 
and Parkinson's disease.

In control cases, lacunes were found in these nuclei in 22 to $39 \%$ of the cases, and only 2 to $8 \%$ of the cases had lacunes bilaterally. In cases with Parkinson's disease the incidence of lacunae in each nucleus is the same as in controls. In patients with vascular parkinsonism, the incidence of unilateral lacunes in each nucleus is the same as in controls, while that of bilateral lacunes is by far much more greater than controls, particularly in the putamen.

This may imply that unilateral involvement of the putamen does not produce parkinsonism, probably due to some sort of compensation: When the putamen is lesioned bilaterally, parkinsonism develops symmetrically.

Involuntary movements due to cerebrovascular lesions in the basal ganglia

Elderly people frequently present various types of involuntary movements such as tremor, choreo-athetosis and oral dyskinesias. One of us (H.T.) studied the relationship between the types of involuntary movements and their responsible lesions in the basal ganglia, using autopsied brains in a geriatric hospital. It was found that tremor was related with putaminal infarctions, choreo-athetosis and oral dyskinesias with lesions in dorsolateral parts of the caudate head.

What was puzzling for us is the relationship between the laterality of the involuntary movements and that of the lesions. According to conventional ideas, unilateral lesion in the basal ganglia gives rise to contralateral involuntary movements and bilateral symptom is induced by bilateral lesions. In our materials, four cases with bilateral putaminal lesions presented either bilateral tremor ( 3 cases) or unilateral tremor. Bilateral lesions may be associated with unilateral symptoms, because it is possible that only lesions on one side may have the effect to produce the symptom. Four cases with unilateral lesions presented bilateral tremor. This seems to be in conflict with conventional ideas.

In patients with choreo-athetoid movements, 5 cases with bilateral lesions in the anterior striatum presented the symptom either bilaterally ( 3 cases) or unilaterally ( 1 case). Out of 7 cases with unilateral lesions in the anterior atriatum, 4 presented choreo-athetoid movements contralaterally. However, the symptom appeared bilaterally in one case, and ipsilaterally in 2 cases.

Review of the literature revealed that choreatic movements associated with ipsilateral striatal lesion is extremely exceptional, but possible. Austregesilo and Gallotti (1924) reported a case in whom hemichorea was associated with involvement of ipsilateral caudate head.

These clinical findings suggest bilateral connections of the basal ganglia and the cortico-striatal pathways. Evidences suggesting bilateral cortico-striatal projections have been accumulating in several animals.

Bilateral regulation of neuronal systems in the basal ganglia has been demonstrated in extensive studies of Glowinski and his collaborators (1984). They implanted halothane-anesthetized cats with several push-pull cannulae so that they could estimate release of newly synthesized tritium-labelled dopamine 
from four structures; nerve terminals in bilateral caudate nucleus and dendrites in bilateral substantia nigra.

They found two patterns in the bilateral effects ; asymmetrical and symmetrical. In asymmetrical effects, unilateral treatments gave rise to opposite effects ; facilitation on ipsilateral nucleus and inhibition on the contralateral nucleus or vice versa. Asymmetrical effects were obtained by infusing dopaminergic drugs into the substantia nigra unilaterally, sensory stimuli, both somatic and visual, and electrical stimulation on cerebellar nuclei and motor thalamic nuclei. In symmetrical effects, unilateral treatments resulted in the same effect, either facilitatory or inhibitory, on both sides of a structure. Symmetrical effects were obtained by unilateral treatments such as infusing potassium, Muscimol and Diazepam, electrical stimulation of intralaminar and midline thalamic nuclei, and cerebral cortex.

These asymmetrical and symmetrical effects were supposed to depend upon the interhemispheric transfer of messages through the thalamic massa intermedia or corpus callosum, because sagittal section of these structures prevented bilateral effects of unilateral treatments.

\section{Cerebellum}

It is generally believed that there is no functional asymmetry in the cerebellum as in the cerebrum, although it may be possible that the right cerebellum is more adopted to skilled movements. Speech disorders in cerebellar diseases usually result from vermian or bilateral hemispheric lesions. Although Lichtenberg and Gilman (1978) proposed dominancy of left cerebellar hemisphere in articulation, Sakai (1980) fail to find such assymetrical localization, and contended that lesions involving superior cerebellar surface, the vermis, and intermediate zones were responsible for speech disorders.

\section{Somatic afferent pathways}

\section{Spinal cord}

The clinical pictures of the lesion comprising a lateral half of the spinal cord is known as Brown-Séquard syndrome. Clinical characterization of this syndrome is somewhat different among textbooks.

For example, Raymond D. Adams (1981) says, "Pain and thermal sensation are affected on the opossite side of the body and proprioceptive sensation is affected on the same side as the lesion. - Tactile sensation is not involved, since the fibers from one side of the body are distributed in tracts (posterior columns and anterior spinothalamic) on both sides of the cord." Brodal(1981) says, "This (Brown-Séquard syndrome) is characterized by a homolateral spastic paralysis below the level of the lesion, _- abolished deep sensibility and sense of discrimination in the same regions, of the contralateral side of the lesion."

It is generally believed that in the spinal cord the pain and temperature pathways enter the cord, ascend two to three segments, and cross the cord anterior 
to the central canal. Accurate touch and two-point discrimination and joint position sense are relayed to the brain in the gracile and cuneate fasciculi on the same side of the spinal cord. If this is the case, spinal hemitransection gives rise to homolateral impairment of touch and joint sensitivity and contralateral impairment of pain and temperature sensation. This is the typical clinical picture described in most textbooks.

Practically however, the typical Brown-Séquard syndrome is relatively rare. Rather, impaired sensitivity of all modalities on the same side of the region is a common feature.

Therefore, the level of the spinal cord where ascending sensory fibers cross may not be so absolute as is generally thought; some fibers for pain and temperature sensation may ascend uncrossed to high cervical cord, or remain uncrossed along their course to the sensory cortex.

\section{Cerebrum}

Bilateral representation for sensation in both hemispheres is supposed from observations on patients with somatosensory cortical lesions. In an acute lesion of the postcentral gyrus, all sensory modalities, both superficial and deep, are impaired. During the course of recovery, sensibity to pain first begins to restore. Later, the sensation to light touch and temperature is regained. However, abolition of the sense of vibration and joint position persists for longer periods.

The rate of recovery of sensory disturbance is different depending upon parts of the body that are involved. Extensive restitution takes place for the face, especially the oral region, larynx and pharynx, and the anogenital regions. The rate of restitution is more rapid in the neck and trunk than in distal parts of the extremities. These parts of the body in which sensory deficits restore faster coincide with the parts receiving bilateral supranuclear motor innervation. Patients with extensive unilateral damage of the cerebral hemisphere often show only mild diminution of sensivity even when they have a complete hemiplegia. These clinical experiences suggest bilateral representaion of sensation in cerebral hemispheres.

Some of the sensory symptoms are particularly related to right hemisphere lesions. Central pain, both thalamic and suprathalamic, is significantly more frequent in the right than in the left hemisphere lesions. Phantom limb in the leg has been reported to be more frequent in the left than in the right leg.

\section{Visual system}

There is a general tendency in vertebrates that the proportion of undecussated optic nerve fibers in the chiasm increases as the species becomes phylogenetically higher, although there are some exceptions. Optic nerves decussate almost completely in non-mammalian vertebrates. In lower mammals each optic tract conveys predominantly fibers from the contralateral eye. In higher primates, including man, the optic tract contains about half of the fibers from each eye. 


\section{Autonomic nervous system}

In general, the autonomic nervous system seems to respond bilaterally to unilaterally applied external stimuli, and autonomic functions seem to be controlled bilaterally by cerebral autonomic centers. For example, pupils on both sides dilate on pinching either side of the neck in ciliospinal reflex. Cheyne-Stokes respiration occurs when cerebral hemispheres are damaged bilaterally. In some instances however an ipsilateral response to unilateral external stimuli occurs, or unilateral cerebral lesions may cause some autonomic dysfunctions. In the latter case, either side of the cerebral hemispheres may play a predominant role in controlling autonomic functions.

\section{Pupillary light response}

When either eye is illuminated, both pupils contract. The constriction of the ipsilateral pupil is termed the direct reaction, and that of the contralateral pupil is termed consensual reaction. It is generally believed that the magnitude of the direct and consensual reflexes are equal.

However, Lowenstein (1954) described a syndrome that he termed alternating contraction anisocoria. In this syndrome, the direct pupillary constriction of the illuminated eye exceeded the consensual constriction. He termed this anisocoria "alternating", because the side of the smaller and larger pupils depended upon the side of illuminated eye (Fig. 2).

Twenty-five years later, Smith et al. studied on this subject using television pupillometry (Smith and Smith 1979 ; Smith et al. 1979). They found that the direct reaction exceeded the consensual reaction in $61(84.7 \%)$ of the 72 normal subjects. The degree of anisocoria was extremely small, with a mean of only $0.075 \mathrm{~mm}$. They classified contraction anisocoria into three patterns. In the bilateral form, the light-induced anisocoria occurred, no matter which eye was stimulated. In one type of unilateral form, illumination of only one side gave a greater response in ipsilateral pupil, while stimulation of the other side resulted in equal pupillary responses. In the second type of unilateral form, the pupillary

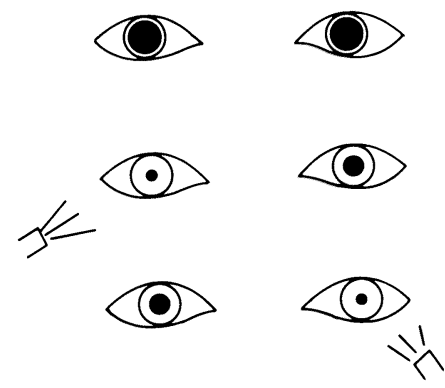

Fig. 2. Alternating contraction amisocoria in which the direct constriction of the illuminated eye exceeds the consensual constriction. 
response of one eye exceeded that of the other eye, whether the response was direct or consensual. This second form of unilateral contraction anisocoria has not been confirmed by Loewenfeld, who believes that the direct light response is always greater than the consensual response (Miller, 1985).

Lowenstein (1954) considered alternating contraction anisocoria a pathologic syndrome, while Smith et al. believed the existence of a small degree of contraction anisocoria in normal individuals. In our experience, contraction anisocoria is often noted in normal subjects even by naked eye observation in clinical practice. When a patient is lying at a window, his pupil on the side of window is often slightly smaller than the other pupil. Pupillary constriction by illumination is often slower and relatively insufficient in consensual than in direct response.

In modern textbooks, the pathway for pupillary light response is postulated as follows. The axons of the ganglion cells in the retina travel intracranially as the optic nerves, optic chiasm, and optic tracts. Before reaching the lateral geniculate body, they branch to send collateral fibers to the pretectal nucleus. Axons of neurons in the pretectal nucleus on both sides pass to the visceral nuclei of the oculomotor complex (Edinger-Westphal nucleus) bilaterally. If this is the case, alternating pupillary response may not occur. Wyatt and Musselman (1981) have postulated the existence of an "extra" pathway from the nasal retina of each eye to the pretectum that produces an "unbalanced" pathway for pulillary responses. Miller (1985), however, dismissed this suggestion.

To account for the alternating anisocoria and benign anisocoria in normal humans (Loewenfeld 1977), somewhere in the pathway of pupillary response should be asymmetrical. For example, uncrossed fibers from the retina to the ipsilateral pretectal nucleus may exceed crossed fibers, or the pathway from the pretectal nucleus to the ipsilateral Edinger-Westphal nucleus may predominate the connection to the contralateral nucleus. Further studies on the anatomical basis of light response is necessary to explain the mechanism of contraction anisocoria.

\section{Cerebral control on micturition}

The uninhibited bladder characterized by urgency, small volume of thresholds and frequency of micturition without residual urine and incontinence is associated with damage of the brain or incomplete interruption of spinal pathway. Abortive micturition contractions not accompanied by a desire to micturate are seen at cystometry. This type of bladder is clinically attributed to release from cortical inhibition which is assumed to descend in the corticospinal tract. Physiologically, however, brainstem facilitation is more likely (Ruch 1960).

Specific frontal lobe areas of the human cerebral cortex, such as superomedial portion of the frontal lobe, the anterior portion of the cingulate gyrus and the corpus callosum play an essential role in the normal micturition reflex. Experimental stimulation in animals suggested that many other areas of the cerebral 
cortex may be involved in micturition as well. Transection experiments have indicated that the net effect of these areas is inhibitory.

We had the impression that urinary symptoms are more frequent with right hemisphere lesions than with left hemisphere lesions, and confirmed this by studying hemiplegic patients after stroke.

135 hemiplegic patients were randomly selected; 68 with right hemiparesis and 67 with left hemiparesis. The proportion of cerebral infarction and cerebral hemorrhage was about the same in both groups. There was no difference in other backgrounds in the two groups. The number of times for daily micturition was documented for 30 days and a mean number of times was calculated for the period. All the patients were asked whether or not they had urinary urgency, urinary incontinence, and urinary retention. A mean frequency of urination of 9 or more was noted in 5 cases with right hemiparesis and 19 cases with left hemiparesis. Urinary urgency was complained by 9 patients with right hemiparesis compared with 24 patients with left hemiparesis $(p<0.01)$. Urinary urgency associated with frequent micturition (9 or more times a day) was noted in 17 cases with left hemiparesis, while absent in 3 patients with right hemiparesis $(p<0.01)$.

Cerebral controls on micturition are both facilitatory and inhibitory. Acute stroke patients often present urinary retention, whereas most patients in chronic periods with bladder disturbance complain urinary urgency and frequent micturition. These symptoms may be due partly to disinhibition of initiation of micturition and partly to inssufficient voiding of urine. The results of our study suggest right cerebral dominancy in controlling bladder functions at least in some people.

In this regard, one of us have reported an interesting case who complained urinary retention and strong urge to micturate only (Kuroiwa et al. 1981). Otherwise, there was no abnormal finding in neurological examination on admission. Cranial CT scan showed an osteoma at the right Rolandic fissure which was confirmed by surgery.

\section{Discussion and Conclusion}

From this brief review, it seems that the unilateral or bilateral representation of the cerebral cortex is a quantitative matter. There is a spectrum from one extreme, where contralateral innervation predominates, to the other extreme, where ipsilateral control is the rule. Bilateral innervation is at the midpoint in this spectrum (Fig. 3).

Supranuclear innervation is usually bilateral for midline structures such as frontal muscles and bulbar muscles, or for controlling symmetrical, synergistic or conjugate movements of paired structures such as arms, legs and eyes. For the sake of safety for functions, bilateral innervation is most appropriate, because even if one hemisphere is injured, the other hemisphere will function for both sides of the body. Therefore, there must be some reasons for the fact that unilateral 

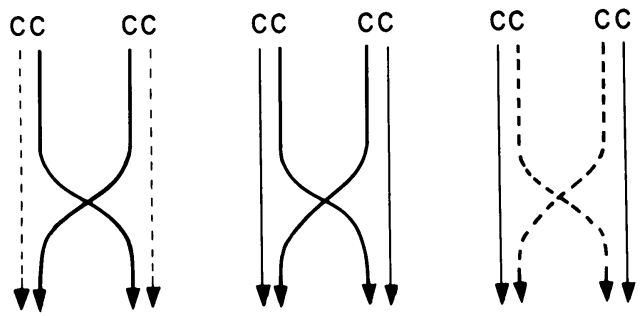

Fig. 3. A spectrum of contralateral (left), bilateral (middle), and ipsilateral (right) representations in cerebral cortex (CC).

innervation generally supercedes for paired structures.

Unilateral innervation may be preferable for each side of the body to execute delicate movements and percept stimuli from the outer world independently. Contralateral innervation is the rule in most instances, while ipsilateral innervation superceds in rare occasions. Whether a function is represented contralaterally or ipsilaterally seems to depend partly upon its relationship with functions of other systems. For example, turning of the head is closely related to conjugate gaze of the eyes. Therefore, the sternocleidomastoid muscle needs to be innervated ipsilaterally.

At a rare extreme, one hemisphere controls somatic or autonomic functions for both sides of the body. Such an asymmetrical and deviated representation of cerebral hemispheres for somatic or autonomic functions parallels that for higher cerebral functions which are represented in specified areas in one hemisphere.

Why, then, are the representations of higher cerebral functions allocated to areas in one hemisphere? Only speculative answers are possible for this question.

During human evolution, the development of unique abilities of language and spacio-temporal recognition required large hemispheric space. Eccles (1981) speculated that this large demand on hemispheric space could be met only by eliminating the redundancy of bilateral representation (Fig. 4-a). If the space is the sole problem, however, the demand can be met by allocating each half of the

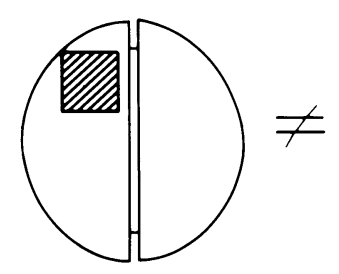

(a)

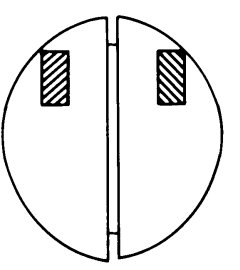

(b)

Fig. 4. The cortical space needed for a function may be allocated either to one hemisphere only (left) or to both hemispheres (a half to each) (right). Hemispheric dominancy for some functions suggests that the space is not the sole problem. The space for a function in one hemisphere and the half space allocated to both hemispheres have different biological meanings. 
space needed for a function to each hemisphere (Fig. 4-b). Consequently, there should be other reasons of deviated representation of specified functions in one hemisphere. Among neuropsychological symptoms, fundamental functions such as memory are represented in both hemisphere, though some cases have been reported to have become amnesic with unilateral temporal lobe lesions. One possible explanation for asymmetrical cerebral representations in higher cerebral functions may be that if the center of a function is represented in both hemispheres, there may be a conflict and confusion between the two centers.

Comparative and evolutional studies on the cortical representation of somatic and autonomic functions may reveal the meanig of different patterns of cerebral representation. Moreover, they may shed light on our better understanding of cortical specialization in higher cerebral functions.

\section{Acknowledgment}

Autopsy data on supranuclear bulbar palsy, vascular parkinsonism, and involuntary movements are based on the works by H.T. in Tokyo Metropolitan Geriatric Center during the period of 1973-1982. He thanks Emeritus prof. Yasuo Toyokura, Prof. Masanori Tomonaga, Dr. Masahiro Yoshimura, Dr. Hiroshi Yamanouchi, Dr. Makoto Ogawa for their close cooperation. We also thank Miss Masako Yamazaki for preparation of the manuscript.

\section{References}

1) Adams, R.D. \& Victor, M. (1981) Principles of Neurology. 2nd ed., McGraw-Hill, New York, p. 114.

2) Austregesilo, A. \& Gallotti, O. (1924) Sur un cas d'hémiparésie et d'hémichorée avec lésion du noyau caudé. Rev. Neurol. (Paris), 1, 41-43.

3) Balegura, S. \& Katz, R.G. (1980) Undecussated innervation to the sternocleidomastoid muscle: A reinstatement. Ann. Neurol., 7, 84-85.

4) Brodal, A. (1957) The Cranial Nerves: Anatomy and Anatomico-Clinical Correlation. Blackwell, Oxford, p. 459.

5) Brodal, A. (1981) Neurological Anatomy in Relation to Clinical Medicine. Oxford University Press, New York, p. 459.

6) Carpenter, M.B. (1972) Core Text of Neuroanatomy. Williams \& Wilkins, Baltimore, pp. 74-75, 81-82.

7) Cogan, D.G. (1956) Neurology of the Ocular Muscles. 2nd ed., Charles C. Thomas Publisher Company, Springfield, pp. 139-148.

8) DeJong, R.M. (1970) The Neurologic Examination. 3rd ed., Harper \& Row, New York, p. 325.

9) Eccles, J.C. (1981) Circumscribed cerebral lesion. In: The Self and Its Brain, edited by K.R. Popper \& J.C. Eccles, Springer International, New York, pp. 334-354.

10) Glowinski, J., Besson, M.J. \& Cheramy, A. (1984) Role of the thalamus in the bilateral regulation of dopaminergic and GABA-ergic neurons in the basal ganglia. In: Functions of the Basal Ganglia, Ciba Foundation Symposium, Pitman, London, pp. $150-160$.

11) Haymaker, W. (1969) Bing's Local Diagnosis in Neurological Diseases. 15th ed., Mosby, St. Louis, p. 181.

12) Haymaker, W. \& Kulenbeck, H. (1978) Disorders of the brain stem and its cranial nerves. In : Clinical Neurology, edited by A.B. Baker \& L.H. Baker, Vol. 3, Harper \& Row, Philadelphia-New York, pp. 2-4. 
13) Holmes, G. (1938) The cerebral integration of ocular movements. Br. Med. J., 2, 107-112.

14) Kameyama, M., Mannen, T. \& Takahashi, K. (1963) Clinicopathological significance of variation of pyramidal decussation. Clin. Neurol., 3, 444-451. (in Japanese with English abstract)

15) Kuroiwa, Y., Furukawa, T. Hiratsuka, H. \& Inaba, Y. (1981) Urinary retention as a sole symptom of intracranial carcified mass at the Rolandic fissure. Ann. Neurol., 10, 61-62.

16) Kuypers, H.G.J.M. (1958) Corticobulbar connections to the pons and lower brain stem in man - an anatomical study. Brain, 81, 364-388.

17) Lichtenberg, R. \& Gilman, S. (1978) Speech disorders in cerebellar diseases. Ann. Neurol., 3, 285-290.

18) Loewenfeld, I.E. (1977) "Simple central" anisocoria: A common condition seldom recognized. Trans. Am. Acad. Ophthal. Otolaryng., 83, 832-839.

19) Lowenstein, O. (1954) Alternating contraction anisocoria. Arch. Neurol. Psychiatr., 72, 742-757.

20) Mayo Clinic (1976) Clinical Examination in Neurology. 4th ed., Saunders, Philadelphia, pp. 66-67.

21) Meadows, J.C. (1973) Dysphagia in unilateral cerebral lesions. J. Neurol. Neurosurg. Psychiatry, 36, 853-860.

22) Miller, N.R. (1985) Walsh and Hoyt's Clinical Neuro-Ophthalmology. 4th ed., Williams Wilkins, Baltimore, pp. 430-432.

23) Netter, F.H. (1958) Ciba Collection of Medical Illustrations: The Nervous System. Ciba Foundation, West Cardwell, pp. 42-43, 47.

24) Nyberg-Hansen, R. \& Rinvik, E. (1963) Some comments on the pyramidal tract, with special reference to its individual variations in man. Acta Neurol. Scand., 39, $1-30$.

25) Patten, J. (1981) Neurological Differential Diagnosis. Harold Starke Ltd., Springer-Verlag, p. 110

26) Peele, T.L. (1961) The Neuroanatomic Basis for Clinical Neurology. 2nd ed., McGraw-Hill, New York, p. 231.

27) Ruch, T.C. (1960) Central control of bladder. In : Handbook of Physiology, Neurophysiology, edited by J. Field \& H.W. Magoun, Vol. 2, Amer. Physiol. Soc., Washington D.C., pp. 1207-1223.

28) Sakai, H. (1980) Cerebellar dysarthria : Historical background and new concept of functional localization. Brain Nerve, 32, 1235-1245. (in Japanese with English abstract)

29) Smith, S.A. \& Smith, S.E. (1979) Light-induced anisocoria in man. J. Physiol. (Lond.), 293, 58.

30) Smith, S.A., Ellis, C.J.K. \& Smith, S.E. (1979) Inequality of the direct and consensual light reflexes in normal subjects. Br. J. Ophthalmol., 63, 523-527.

31) Warwick, R. (1953) Representation of the extra-ocular muscles in the oculomotor nuclei of the monkey. J. Comp. Neurol., 98, 449-503.

32) Wyatt, H.J. \& Musselman, J.F. (1981) Pupillary light reflex in humans : Evidence for an unbalances pathway from nasal retina, and for signal cancellation in brainstem. Vision Res., 21, 513-525.

33) Zenner, P. (1898) Ein Fall von Hirngeschwulst in der linken motorische Sphäre, linkseitiger Lahmung. Abwesentheit der Pyramidenkreuzung. Neurol. Zentralbld., 17, 202. (cited from Holmes 1938) 